\title{
Cascade of instabilities in the classical limit of the Berenstein-Maldacena- Nastase matrix model
}

\author{
Minos Axenides $\odot,{ }^{1, *}$ Emmanuel Floratos, ${ }^{1,2, \dagger}$ Dimitrios Katsinis $\odot,{ }^{1, \ddagger}$ and Georgios Linardopoulos $\oplus^{1, \S}$ \\ ${ }^{1}$ Institute of Nuclear and Particle Physics, \\ National Centre for Scientific Research, “Demokritos," 153 10, Agia Paraskevi, Greece \\ ${ }^{2}$ Department of Nuclear and Particle Physics, National and Kapodistrian University of Athens, \\ 157 84, Athens, Greece
}

(Received 13 September 2021; accepted 6 October 2021; published 5 November 2021)

\begin{abstract}
We study the leading- (LO) and the next-to-leading-order (NLO) stability of multipole perturbations for a static dielectric M2-brane with spherical topology in the 11-dimensional maximally supersymmetric plane-wave background. We observe a cascade of instabilities that originates from the dipole $(j=1)$ and quadrupole $(j=2)$ sectors (the only unstable sectors of the LO) and propagates toward all the multipoles of the NLO.
\end{abstract}

DOI: $10.1103 /$ PhysRevD.104.106002

\section{INTRODUCTION}

According to our current understanding of the black hole $(\mathrm{BH})$ information paradox, the chaotic dynamics of the microscopic degrees of freedom that are present on $\mathrm{BH}$ horizons is an indispensable aspect of its resolution [1]. In the present paper, we make an analogy with the $\mathrm{BH}$ membrane paradigm [2,3] and attempt to describe the dynamics of $\mathrm{BH}$ horizons by means of relativistic membranes inside plane-wave spacetimes. In the context of $\mathrm{M}$ theory, these membranes arise from the BerensteinMaldacena-Nastase (BMN) matrix model in the limit of large matrix dimensions $[4,5]$. Our goal is to study the emergence of classical chaos at both the leading (LO) and the next-to-leading order (NLO) of perturbation theory for various spherical membranes, by using methods from chaos theory and dynamical systems.

Our previous findings [6-8] imply that the various modes of multipole perturbations are coupled to each other. This effect is responsible for the propagation (or the cascade) of dipole and quadrupole instabilities from LO perturbation theory to higher-order multipoles (which are stable to LO). There is a striking similarity between this cascade of instabilities and the avalanche phenomenon (i.e., the

\footnotetext{
*axenides@inp.demokritos.gr

mflorato@phys.uoa.gr

*dkatsinis@phys.uoa.gr

glinard@inp.demokritos.gr
}

Published by the American Physical Society under the terms of the Creative Commons Attribution 4.0 International license. Further distribution of this work must maintain attribution to the author(s) and the published article's title, journal citation, and DOI. Funded by SCOAP ${ }^{3}$. breaking of large vortices into smaller ones) that characterizes turbulent flows in hydrodynamics (see e.g., [9]).

Our paper is organized as follows. In Sec. II we review the $S O(3)$ symmetric ansatz which is our main object of study. In Sec. III we set up and carry out the LO perturbative analysis for this system, filling out and supplying many details that were omitted in our previous works. In Sec. IV we proceed to the study of higher-order perturbations that lead to the instability cascade phenomenon. An illustrative example of instability cascades can be found in Sec. V. A brief discussion of our findings as well as the conclusions have been included in Sec. VI.

\section{SETUP}

One of the most remarkable properties of plane-wave spacetimes is that they can be obtained from any given metric via the Penrose limiting procedure [10], which consists in blowing up the spacetime around null geodesics (effectively "zooming in" to them). As it turns out, plane waves preserve the supersymmetries of the original background, so that the maximally supersymmetric spacetimes $\operatorname{AdS}_{4 / 7} \times \mathrm{S}^{7 / 4}$ of 11-dimensional supergravity give rise to the maximally supersymmetric plane-wave solution [11]:

$$
\begin{gathered}
d s^{2}=-2 d x^{+} d x^{-}+\sum_{i=1}^{3} d x_{i} d x_{i}+\sum_{k=1}^{6} d y_{k} d y_{k} \\
-\left[\frac{\mu^{2}}{9} \sum_{i=1}^{3} x_{i} x_{i}+\frac{\mu^{2}}{36} \sum_{k=1}^{6} y_{k} y_{k}\right] d x^{+} d x^{+}, \\
F_{123+}=\mu,
\end{gathered}
$$


where $F_{\mu \nu \rho \sigma}$ is the field strength tensor of 11-dimensional supergravity.

The Hamiltonian of a bosonic relativistic membrane in the 11-dimensional maximally supersymmetric plane-wave background (1)-(2) reads in the so-called light-cone gauge $\left(x^{+} \equiv \tau\right)$

$$
\begin{aligned}
H= & \frac{T}{2} \int_{\Sigma} d^{2} \sigma\left[p_{x}^{2}+p_{y}^{2}+\frac{1}{2}\left\{x_{i}, x_{k}\right\}^{2}+\frac{1}{2}\left\{y_{i}, y_{k}\right\}^{2}\right. \\
& \left.+\left\{x_{i}, y_{k}\right\}^{2}+\frac{\mu^{2} x^{2}}{9}+\frac{\mu^{2} y^{2}}{36}-\frac{\mu}{3} \epsilon_{i k l}\left\{x_{i}, x_{k}\right\} x_{l}\right],
\end{aligned}
$$

where $T$ is the membrane tension and the indices of the coordinates $x$ and $y$ run from 1 to 3 and 1 to 6 , respectively. The corresponding dynamics is described by the Gauss law constraint

$$
\left\{\dot{x}_{i}, x_{i}\right\}+\left\{\dot{y}_{k}, y_{k}\right\}=0
$$

and the equations of motion

$$
\begin{aligned}
\ddot{x}_{i}= & \left\{\left\{x_{i}, x_{k}\right\}, x_{k}\right\}+\left\{\left\{x_{i}, y_{k}\right\}, y_{k}\right\}-\frac{\mu^{2}}{9} x_{i} \\
& +\frac{\mu}{2} \epsilon_{i k l}\left\{x_{k}, x_{l}\right\}, \\
\ddot{y}_{i}= & \left\{\left\{y_{i}, y_{k}\right\}, y_{k}\right\}+\left\{\left\{y_{i}, x_{k}\right\}, x_{k}\right\}-\frac{\mu^{2}}{36} y_{i} .
\end{aligned}
$$

It can be shown that the regularized light-cone supermembrane (3) is described by the BMN matrix model [12]. Equivalently, the Hamiltonian (3) describes the continuum limit of the BMN matrix model [5]. In the absence of background flux $(\mu=0)$, the plane wave (1)-(2) becomes flat 11-dimensional spacetime and the BMN matrix model reduces to the matrix model of Banks, Fischler, Shenker and Susskind [13].

The presence of a nonzero 4-form field strength $F_{\mu \nu \rho \sigma}$ in the plane-wave background (2) induces repulsive flux terms in the membrane effective potential. The potential also has attractive quartic and quadratic terms. These are respectively induced by the self-interaction terms of the M2-brane action and the mass terms of the plane-wave metric (1). The competition of attractive and repulsive forces gives rise to stable dielectric membrane solutions that are named after Myers who studied a similar effect [14].

A static dielectric Myers solution of spherical topology that lives exclusively in the $S O(3)$ symmetric subspace of the background (1)-(2) can be obtained from the following ansatz :

\footnotetext{
${ }^{1}$ See [8] for a generalized form of this solution. A similar ansatz was studied in [15], although the prototype was probably introduced a long time ago [16].
}

$x_{i}=\mu u_{i} e_{i}, \quad i=1,2,3, \quad y_{k}=0, \quad k=1, \ldots 6$,

where the three coordinates of the unit sphere,

$$
\left(e_{1}, e_{2}, e_{3}\right)=(\cos \phi \sin \theta, \sin \phi \sin \theta, \cos \theta),
$$

satisfy the $\mathfrak{g} \mathfrak{v}(3)$ Poisson bracket algebra and are orthonormal:

$$
\left\{e_{a}, e_{b}\right\}=\epsilon_{a b c} e_{c}, \quad \int e_{a} e_{b} d^{2} \sigma=\frac{4 \pi}{3} \delta_{a b} .
$$

The membrane effective potential that arises when the ansatz (7) is inserted into the Hamiltonian (3) is the generalized 3d Hénon-Heiles potential [17]:

$$
\begin{aligned}
V_{\text {eff }}= & \frac{2 \pi T \mu^{4}}{3}\left[u_{1}^{2} u_{2}^{2}+u_{2}^{2} u_{3}^{2}+u_{1}^{2} u_{3}^{2}\right. \\
& \left.+\frac{1}{9}\left(u_{1}^{2}+u_{2}^{2}+u_{3}^{2}\right)-2 u_{1} u_{2} u_{3}\right] .
\end{aligned}
$$

Equation (10) has exactly nine critical points [7]. These are

$$
\mathbf{u}_{0}=0, \quad \mathbf{u}_{1 / 6}=\frac{1}{6}(1,1,1), \quad \mathbf{u}_{1 / 3}=\frac{1}{3}(1,1,1),
$$

as well as six more extrema that are obtained from $\mathbf{u}_{1 / 6}$ and $\mathbf{u}_{1 / 3}$ by flipping the signs of two of their components. The discrete symmetry group of the nine extremal points of (10) is the tetrahedral group $T_{d}$. This discrete symmetry is shared by the equations of motion (4) and (5). To see this, apply separate reflections to the coordinates $x_{i} \rightarrow \epsilon_{i} x_{i}\left(\epsilon_{i}= \pm 1\right)$ and note that the flux term implies the constraint $\epsilon_{1} \epsilon_{2} \epsilon_{3}=1$.

In sum, the potential (10) has degenerate minima at $\mathbf{u}_{0}$ (a pointlike membrane) and $\mathbf{u}_{1 / 3}$ (the Myers dielectric sphere) and a saddle point at $\mathbf{u}_{1 / 6}$ (unstable static sphere):

$V_{\text {eff }}(0)=V_{\text {eff }}\left(\frac{1}{3}\right)=0, \quad V_{\text {eff }}\left(\frac{1}{6}\right)=\frac{2 \pi T \mu^{4}}{6^{4}}$

The radial stability analysis of the solution (7) around the extremal points (11) was carried out in [6] where it was shown that $\mathbf{u}_{0}$ and $\mathbf{u}_{1 / 3}$ are radially stable, whereas $\mathbf{u}_{1 / 6}$ is a radially unstable point.

\section{LEADING-ORDER PERTURBATIONS}

The study of angular perturbations around the spherically symmetric extrema (11) was performed in [7]. Below we revisit the main points of the analysis and restate its main results by supplying certain new details.

The static ansatz $x_{i}(\tau)=\mu u_{0} e_{i}$ is constructed from the $S O(3)$ extrema $u_{0}=\{0,1 / 6,1 / 3\}$. It satisfies (5) for $y_{k}=0$ : 


$$
\ddot{x}_{i}=\left\{\left\{x_{i}, x_{k}\right\}, x_{k}\right\}-\frac{\mu^{2}}{9} x_{i}+\frac{\mu}{2} \epsilon_{i k l}\left\{x_{k}, x_{l}\right\}
$$

and corresponds to the initial conditions $x_{i}(0)=\mu x_{i}^{0}$ and $\dot{x}_{i}(0)=0$, at $\tau=0$. The perturbative analysis which was performed in the paper [7] is based on the work of Lyapunov (details can be found in many standard textbooks, e.g., [18]). Consider an infinitesimal variation of the initial conditions (for $i=1,2,3$ )

$$
x_{i}(0)=\mu\left(x_{i}^{0}+\varepsilon \delta x_{i}(0)\right), \quad \dot{x}_{i}(0)=\mu \varepsilon \delta \dot{x}_{i}(0) .
$$

The perturbation of the initial conditions (14) induces an $\varepsilon$ dependence in the solution $x_{i}(\tau, \varepsilon)$. In general, $x_{i}(\tau, \varepsilon)$ affords a series expansion in $\varepsilon$ :

$$
x_{i}=\mu\left(x_{i}^{0}+\sum_{n=1}^{\infty} \varepsilon^{n} \delta x_{i}^{(n)}\right), \quad i=1,2,3 .
$$

Inserting the perturbation (15) into the equations of motion (13), we obtain to leading order $\varepsilon$

$$
\begin{aligned}
\delta \ddot{x}_{i}= & \left\{\left\{\delta x_{i}, x_{j}^{0}\right\}, x_{j}^{0}\right\}+\left\{\left\{x_{i}^{0}, \delta x_{j}\right\}, x_{j}^{0}\right\} \\
& +\left\{\left\{x_{i}^{0}, x_{j}^{0}\right\}, \delta x_{j}\right\}-\frac{1}{9} \delta x_{i}+\epsilon_{i j k}\left\{\delta x_{j}, x_{k}^{0}\right\},
\end{aligned}
$$

where, from now on, we switch to the dimensionless time $t \equiv \mu \tau$. For simplicity we have also set $\delta x_{i}^{(1)} \equiv \delta x_{i}$. The Gauss law constraint (4) becomes

$$
\left\{\delta \dot{x}_{i}, x_{i}^{0}\right\}=0 .
$$

It can be shown [7] that if (17) is satisfied at $t=0$, then the perturbation equations (16) guarantee its validity at all times $t$. Following the works [19], we expand $\delta x$ in spherical harmonics:

$$
\delta x_{i}=\sum_{j, m} \eta_{i}^{j m}(t) Y_{j m}(\theta, \phi),
$$

where $\delta x$ must be real, so that the fluctuation modes $\eta_{i}^{j m}(t)$ satisfy

$$
\eta_{i}^{j m *}(t)=(-1)^{m} \eta_{i}^{j(-m)}(t)
$$

By using the property of spherical harmonics,

$$
\begin{aligned}
\left\{e_{i}, Y_{j m}(\theta, \phi)\right\} & =-i \hat{J}_{i}^{(j)} Y_{j m}(\theta, \phi) \\
& =-i \sum_{m^{\prime}}\left(J_{i}\right)_{m^{\prime} m}^{(j)} Y_{j m^{\prime}}(\theta, \phi),
\end{aligned}
$$

where $\hat{J}_{i}^{(j)}$ is the spin- $j$ angular momentum operator and $\left(J_{i}\right)_{m m^{\prime}}^{(j)}$ the $2 j+1$-dimensional (spin- $j$ ) matrix representation of $\mathfrak{g} \mathfrak{t}(2)$, we can show that the fluctuation modes $\eta_{i}$ satisfy the following equations of motion:

$$
\ddot{\eta}_{i}+\omega_{3}^{2} \eta_{i}=u_{0}^{2} T_{i k} \eta_{k}+u_{0} Q_{i k} \eta_{k} .
$$

In (21), we have omitted the indices $j$ and $m$; we have summed the repeated indices and have used the definitions

$$
\begin{aligned}
\omega_{3}^{2} \equiv u_{0}^{2} \mathbf{J}^{2}+\frac{1}{9}, & T_{i k} \equiv J_{i} J_{k}-2 i \epsilon_{i k l} J_{l}, \\
Q_{i k} & \equiv i \epsilon_{i k l} J_{l},
\end{aligned}
$$

where $\mathbf{J}^{2}=j(j+1)$. Equation (21) can be written in a compact form as follows:

$$
\ddot{H}+\mathcal{K} \cdot H=0, \quad \mathcal{K} \equiv \omega_{3}^{2}-u_{0}^{2} T-u_{0} Q,
$$

where $H, Q$ and $T$ refer to the $3 \times(2 j+1)$-dimensional representations of $\eta_{i}^{j m}, Q_{i k}$ and $T_{i k}$, respectively:

$$
\begin{gathered}
H=\left(\begin{array}{c}
\eta_{x}^{j m} \\
\eta_{y}^{j m} \\
\eta_{z}^{j m}
\end{array}\right), \quad Q=i\left(\begin{array}{ccc}
0 & J_{z} & -J_{y} \\
-J_{z} & 0 & J_{x} \\
J_{y} & -J_{x} & 0
\end{array}\right), \\
T=\left(\begin{array}{ccc}
J_{x}^{2} & J_{x} J_{y}-2 i J_{z} & J_{x} J_{z}+2 i J_{y} \\
J_{y} J_{x}+2 i J_{z} & J_{y}^{2} & J_{y} J_{z}-2 i J_{x} \\
J_{z} J_{x}-2 i J_{y} & J_{z} J_{x}+2 i J_{x} & J_{z}^{2}
\end{array}\right) .
\end{gathered}
$$

The $2 j+1$ and the $3 \times(2 j+1)$-dimensional representations (21) and (24) are in many ways inequivalent. Many important details of the analysis that follows depend crucially on which of the two representations is being used.

\section{A. Eigenvalues}

In order to solve (24) we set $H=e^{i \lambda t} \xi$. We are led to the following eigenvalue problem in the $3 \times(2 j+1)$ dimensional space:

$$
\left(-\lambda^{2}+\omega_{3}^{2}-u_{0}^{2} T-u_{0} Q\right) \cdot \xi=0, \quad \xi \equiv\left(\xi_{i}^{j m}\right) .
$$

We further introduce the $(2 j+1) \times(2 j+1)$ matrices

$$
\begin{aligned}
P_{i k} \equiv & \frac{1}{j(j+1)} J_{i} J_{k}, \\
R_{i k}^{ \pm} \equiv & \frac{1}{2 j+1}\left[\frac{1}{2}(2 j+1 \mp 1) \cdot\left(\delta_{i k} \times I-P_{i k}\right)\right. \\
& \left. \pm\left(\delta_{i k} \times I-Q_{i k}\right)\right],
\end{aligned}
$$

which form a complete and orthogonal set of projection operators (i.e., idempotent and Hermitian). In the $3 \times(2 j+1)$-dimensional space, 


$$
\begin{aligned}
& P^{2}=P=P^{\dagger}, \quad R_{ \pm}^{2}=R_{ \pm}=R_{ \pm}^{\dagger}, \\
& P \cdot R^{ \pm}=R^{+} \cdot R^{-}=0, \\
& P+R^{+}+R^{-}=I,
\end{aligned}
$$

where $I$ is the $3(2 j+1) \times 3(2 j+1)$ unit matrix. It turns out that the matrices $T$ and $Q$ are also Hermitian and can be expressed in terms of the projectors $P$ and $R_{ \pm}$:

$$
\begin{aligned}
& T=[j(j+1)-2] P+2 j R_{+}-2(j+1) R_{-}, \\
& Q=P-j R_{+}+(j+1) R_{-} .
\end{aligned}
$$

The advantage of this decomposition is that we can immediately read the eigenvalues of the matrices $T$ and $Q$ (along with their degeneracies) in each of the projective spaces $P, R_{ \pm}$. Plugging (33) and (34) into (27) we are led to

$$
\begin{aligned}
\left(\omega_{3}^{2}-\lambda^{2}\right) \xi= & {\left[\left(u_{0}^{2}[j(j+1)-2]+u_{0}\right) P+j u_{0}\right.} \\
& \left.\cdot\left(2 u_{0}-1\right) R_{+}-(j+1) u_{0}\left(2 u_{0}-1\right) R_{-}\right] \xi
\end{aligned}
$$

which we then multiply with the projectors $P$ and $R_{ \pm}$in order to obtain the eigenvalues $\lambda$ :

$$
\begin{aligned}
& \lambda_{P}^{2}=2\left(u_{0}-\frac{1}{3}\right)\left(u_{0}-\frac{1}{6}\right), \\
& \lambda_{+}^{2}=j(j-1) u_{0}^{2}+j u_{0}+\frac{1}{9}, \\
& \lambda_{-}^{2}=(j+1)(j+2) u_{0}^{2}-(j+1) u_{0}+\frac{1}{9} .
\end{aligned}
$$

The degeneracies of the eigenvalues $\lambda_{P, \pm}^{2}$ are equal to the dimensionalities of the corresponding projectors $P$ and $R_{ \pm}$, i.e., $d_{+}=2 j+3, d_{P}=2 j+1$, and $d_{-}=2 j-1$. For each of the critical points in (11) we find

$$
\begin{aligned}
\mathbf{u}_{0}: \quad \lambda_{P}^{2} & =\lambda_{ \pm}^{2}=\frac{1}{9}, \\
\mathbf{u}_{1 / 6}: \quad \lambda_{P}^{2} & =0, \quad \lambda_{+}^{2}=\frac{1}{36}(j+1)(j+4), \\
\lambda_{-}^{2} & =\frac{j(j-3)}{36}, \\
\mathbf{u}_{1 / 3}: & \lambda_{P}^{2}=0, \quad \lambda_{+}^{2}=\frac{1}{36}(j+1)^{2}, \quad \lambda_{-}^{2}=\frac{j^{2}}{9},
\end{aligned}
$$

which coincide with the eigenvalues that were found in [5] for the BMN matrix model. Note that only the $R_{-}$sector of $\mathbf{u}_{1 / 6}$ is unstable for $j=1,2$ ( $\lambda_{-}$is purely imaginary). These instabilities are related to the existence of a separatrix in the corresponding phase diagram (see e.g., [6]).

The general solution of (24) can be written in the $3 \times(2 j+1)$-dimensional space as follows:

$$
\begin{aligned}
H(t)= & e^{i \lambda_{P} t} \xi_{P}+e^{-i \lambda_{P} t} \tilde{\xi}_{P}+e^{i \lambda_{+} t} \xi_{+}+e^{-i \lambda_{+} t} \tilde{\xi}_{+} \\
& +e^{i \lambda_{-} t} \xi_{-}+e^{-i \lambda_{-} t} \tilde{\xi}_{-},
\end{aligned}
$$

where $\xi_{A}, \tilde{\xi}_{A}, A \equiv\{P, \pm\}$ are generic $3 \times(2 j+1)$-dimensional vectors of the subspaces $P$ and $R_{ \pm}$. These are naturally expressed as linear combinations of the corresponding eigenvectors $|P, \pm\rangle$. They are determined by the initial values of $H$ and $\dot{H}$ at $t=0$ and the leading order of the Gauss law constraint [see (59) below].

\section{B. Eigenvectors}

Calculating the square of the matrix $Q$ [defined in (23) above] by using the decomposition (33)-(34), we find that the projection operators $P$ and $R_{ \pm}$can be expressed in terms of $Q$ as follows:

$$
\begin{aligned}
P= & I+\frac{Q-Q^{2}}{j(j+1)}, \quad R_{ \pm}=\frac{1}{2 j+1}\left[\frac{1}{2}(2 j+1 \mp 1)\right. \\
& \left.\cdot \frac{Q^{2}-Q}{j(j+1)} \pm(I-Q)\right] .
\end{aligned}
$$

This implies that the eigenvectors of $P$ and $R_{ \pm}$are fully determined by those of $Q$.

Before going on to derive the precise expressions of these eigenvectors, let us note that $Q$ is just the spin-orbit coupling operator of the orbital angular momentum $L=1$ with the spin angular momentum $J=j$. To see why this is so, consider the so-called adjoint representation of $S U(2)$ in which the three components of the orbital angular momentum $L=1 \mathrm{read}$

$$
\begin{aligned}
& \left(L_{i}\right)_{k l}=i \epsilon_{i l k} \Rightarrow L_{x}=\left(\begin{array}{ccc}
0 & 0 & 0 \\
0 & 0 & -i \\
0 & i & 0
\end{array}\right), \\
& L_{y}=\left(\begin{array}{ccc}
0 & 0 & i \\
0 & 0 & 0 \\
-i & 0 & 0
\end{array}\right), \quad L_{z}=\left(\begin{array}{ccc}
0 & -i & 0 \\
i & 0 & 0 \\
0 & 0 & 0
\end{array}\right) \text {, }
\end{aligned}
$$

and $Q$ indeed takes the form of spin-orbit coupling:

$$
Q_{i k}=\left(L_{l}\right)_{k i} J_{l} \Leftrightarrow Q=-L_{i} \otimes J_{i}
$$

or, in terms of the raising and lowering operators $L_{ \pm} \equiv$ $L_{x} \pm i L_{y}$ and $J_{ \pm} \equiv J_{x} \pm i J_{y}$, 


$$
Q=-\frac{1}{2}\left(L_{+} \otimes J_{-}\right)-\frac{1}{2}\left(L_{-} \otimes J_{+}\right)-L_{z} \otimes J_{z}
$$

The three components of the total angular momentum $\mathbf{J}_{T}=\mathbf{L}+\mathbf{J}$, for $J=j, L=1$, are given by

$$
J_{T}^{i}=J_{i} \otimes I_{3}+I_{2 j+1} \otimes L_{i}
$$

and, explicitly,

$$
\begin{aligned}
J_{T}^{x} & =\left(\begin{array}{ccc}
J_{x} & 0 & 0 \\
0 & J_{x} & -i I \\
0 & i I & J_{x}
\end{array}\right), \quad J_{T}^{y}=\left(\begin{array}{ccc}
J_{y} & 0 & i I \\
0 & J_{y} & 0 \\
-i I & 0 & J_{y}
\end{array}\right) \\
J_{T}^{z} & =\left(\begin{array}{ccc}
J_{z} & -i I & 0 \\
i I & J_{z} & 0 \\
0 & 0 & J_{z}
\end{array}\right)
\end{aligned}
$$

while the square of the total angular momentum is

$$
\mathbf{J}_{T}^{2}=(j(j+1)+2) I_{3(2 j+1)}-2 Q,
$$

so that its eigenstates

$$
|j+1, m ; j, 1\rangle, \quad|j, m ; j, 1\rangle, \quad|j-1, m ; j, 1\rangle
$$

will obviously also diagonalize the spin-orbit coupling operator $Q$.

By directly diagonalizing the matrix $Q$ in (46) or, equivalently, by means of the standard Clebsch-Gordan analysis (see e.g., [20]) we find

$$
\begin{aligned}
|j+1, m ; j, 1\rangle= & \sqrt{\frac{(j+m)(j+m+1)}{2(j+1)(2 j+1)}} \cdot|1,1\rangle|j, m-1\rangle \\
& +\sqrt{\frac{(j+1)^{2}-m^{2}}{(j+1)(2 j+1)}} \cdot|1,0\rangle|j, m\rangle \\
& +\sqrt{\frac{(j-m)(j-m+1)}{2(j+1)(2 j+1)}} \cdot|1,-1\rangle|j, m+1\rangle,
\end{aligned}
$$

$$
\begin{aligned}
|j, m ; j, 1\rangle= & -\sqrt{\frac{(j+m)(j-m+1)}{2 j(j+1)}} \cdot|1,1\rangle|j, m-1\rangle \\
& +\frac{m}{\sqrt{j(j+1)}} \cdot|1,0\rangle|j, m\rangle \\
& +\sqrt{\frac{(j-m)(j+m+1)}{2 j(j+1)}} \cdot|1,-1\rangle|j, m+1\rangle,
\end{aligned}
$$

$$
\begin{aligned}
|j-1, m ; j, 1\rangle= & \sqrt{\frac{(j-m)(j-m+1)}{2 j(2 j+1)}} \cdot|1,1\rangle|j, m-1\rangle \\
& -\sqrt{\frac{j^{2}-m^{2}}{j(2 j+1)}} \cdot|1,0\rangle|j, m\rangle \\
& +\sqrt{\frac{(j+m)(j+m+1)}{2 j(2 j+1)}} \cdot|1,-1\rangle|j, m+1\rangle .
\end{aligned}
$$

Acting with the spin-orbit coupling operator $Q$ on the Clebsch-Gordan system (51)-(53), we obtain the following eigenvalues:

$$
\begin{aligned}
& Q \cdot|j+1, m ; j, 1\rangle=-j|j+1, m ; j, 1\rangle, \\
& Q \cdot|j, m ; j, 1\rangle=|j, m ; j, 1\rangle, \\
& Q \cdot|j-1, m ; j, 1\rangle=(j+1)|j-1, m ; j, 1\rangle .
\end{aligned}
$$

Furthermore, by plugging the eigenstates (51)-(53) into the expressions (43) that give the projectors $P$ and $R_{ \pm}$in terms of the spin-orbit coupling operator $Q$, we find that the eigenstates $|j, m ; j, 1\rangle$ span the subspace of the projector $P$, while the eigenstates $|j \pm 1, m ; j, 1\rangle$ span the subspaces of the projectors $R_{ \pm}$, i.e.,

$$
|P\rangle=|j, m ; j, 1\rangle, \quad| \pm\rangle=|j \pm 1, m ; j, 1\rangle .
$$

\section{Gauss law constraint (LO)}

Plugging the solution (7) and the perturbative expansion (18) into the Gauss law constraint (17) we obtain, to LO in perturbation theory,

$$
\begin{aligned}
\sum_{j, m} \dot{\eta}_{i}^{j m}\left\{Y_{j m}, e_{i}\right\} & =\sum_{j, m, m^{\prime}} \dot{\eta}_{i}^{j m}\left(J_{i}\right)_{m^{\prime} m}^{(j)} Y_{j m^{\prime}}=0 \\
& \Rightarrow \sum_{m} \dot{\eta}_{i}^{j m}\left(J_{i}\right)_{m^{\prime} m}^{(j)}=0
\end{aligned}
$$

since the spherical harmonics $Y_{j m}$ form an orthonormal basis. Multiplying with $J_{k}^{(j)}$ and using the definition (28) of the projector $P$ we find

$$
\begin{aligned}
\sum_{m, m^{\prime}} \dot{\eta}_{i}^{j m}\left(J_{k}\right)_{m^{\prime \prime} m^{\prime}}^{(j)}\left(J_{i}\right)_{m^{\prime} m}^{(j)} & =0 \\
\Rightarrow \sum_{m^{\prime}}\left(P_{i k}\right)_{m m^{\prime}}^{(j)} \dot{\eta}_{k}^{j m^{\prime}} & =0 .
\end{aligned}
$$

Equation (59) constrains the generic form (42) of the LO perturbations $\eta_{i}^{j m}$, which in principle span all the three sectors $P$ and $R_{ \pm}$. 
Because of the Gauss law constraint (59), $\eta_{i}^{j m}$ is forced to live exclusively inside the sectors $R_{ \pm}$at all the critical points (39)-(41). However, it receives an additional contribution from the zero eigenvalue eigenstate $\xi_{P}$ of the $P$ sector at the critical points $\mathbf{u}_{1 / 6}$ and $\mathbf{u}_{1 / 3}$.

\section{HIGHER-ORDER PERTURBATIONS}

Let us now study the perturbative expansion (15) beyond the leading order. The initial conditions for the perturbations $\delta x_{i}^{(n)}$ and their derivatives are determined from the initial conditions of the complete solution (15). They all vanish at $t=0$ :

$$
\delta x_{i}^{(n)}(0)=\delta \dot{x}_{i}^{(n)}(0)=0, \quad n=2,3, \ldots
$$

unless $n=0,1$. To obtain the equations of motion for the perturbations, we plug the series (15) into the $S O(3)$ equations of motion (13). We are led to

$$
\begin{aligned}
\delta \ddot{x}_{i}^{(n)}= & \left\{\left\{\delta x_{i}^{(n)}, x_{k}^{(0)}\right\}, x_{k}^{(0)}\right\}+\left\{\left\{x_{i}^{(0)}, \delta x_{k}^{(n)}\right\}, x_{k}^{(0)}\right\}+\left\{\left\{x_{i}^{(0)}, x_{k}^{(0)}\right\}, \delta x_{k}^{(n)}\right\}-\frac{1}{9} \delta x_{i}^{(n)}+\epsilon_{i k l}\left\{x_{k}^{(0)}, \delta x_{l}^{(n)}\right\} \\
& +\sum_{p=1}^{n-1}\left[\left\{\left\{x_{i}^{(0)}, \delta x_{k}^{(n-p)}\right\}, \delta x_{k}^{(p)}\right\}+\left\{\left\{\delta x_{i}^{(n-p)}, x_{k}^{(0)}\right\}, \delta x_{k}^{(p)}\right\}+\left\{\left\{\delta x_{i}^{(n-p)}, \delta x_{k}^{(p)}\right\}, x_{k}^{(0)}\right\}\right. \\
& \left.+\frac{1}{2} \epsilon_{i k l}\left\{\delta x_{k}^{(n-p)}, \delta x_{l}^{(p)}\right\}\right]+\sum_{p=1}^{n-1} \sum_{q=1}^{p-1}\left\{\left\{\delta x_{i}^{(n-p)}, \delta x_{k}^{(p-q)}\right\}, \delta x_{k}^{(q)}\right\} .
\end{aligned}
$$

As before, we proceed to expand the perturbations in spherical harmonics:

$$
\delta x_{i}^{(n)}=\sum_{j, m} \eta_{i}^{n j m}(t) Y_{j m}(\theta, \phi), \quad \eta_{i}^{n j m}(0)=0,
$$

which, in addition to the reality condition (19) and the property (20), obey

$$
\left\{Y_{\alpha}(\theta, \phi), Y_{\beta}(\theta, \phi)\right\}=f_{\alpha \beta}^{\gamma} Y_{\gamma}(\theta, \phi),
$$

where $f_{\alpha \beta}^{\gamma}$ are the structure constants of the area-preserving symmetry of the Hamiltonian (3) and $\alpha \equiv j m, \beta \equiv j^{\prime} m^{\prime}$, and $\gamma \equiv j^{\prime \prime} m^{\prime \prime}$. The structure constants $f_{\alpha \beta}^{\gamma}$ can be computed by means of a closed formula that is valid for all values of the spin quantum numbers $\alpha, \beta$, and $\gamma$. The expressions for the structure constants $f_{1 m, \beta}^{\gamma}$ and $f_{2 m, \beta}^{\gamma}$ can be found in the Appendix.

\section{A. Next-to-leading-order perturbations}

For $n=2$, it is easy to show that the higher-order perturbation equations (61) become

$$
\begin{aligned}
\delta \ddot{x}_{i}^{(2)}= & \left\{\left\{\delta x_{i}^{(2)}, x_{k}^{(0)}\right\}, x_{k}^{(0)}\right\}+\left\{\left\{x_{i}^{(0)}, \delta x_{k}^{(2)}\right\}, x_{k}^{(0)}\right\}+\left\{\left\{x_{i}^{(0)}, x_{k}^{(0)}\right\}, \delta x_{k}^{(2)}\right\}-\frac{1}{9} \delta x_{i}^{(2)}+\epsilon_{i k l}\left\{x_{k}^{(0)}, \delta x_{l}^{(2)}\right\} \\
& +\left[\left\{\left\{x_{i}^{(0)}, \delta x_{k}^{(1)}\right\}, \delta x_{k}^{(1)}\right\}+\left\{\left\{\delta x_{i}^{(1)}, x_{k}^{(0)}\right\}, \delta x_{k}^{(1)}\right\}+\left\{\left\{\delta x_{i}^{(1)}, \delta x_{k}^{(1)}\right\}, x_{k}^{(0)}\right\}+\frac{1}{2} \epsilon_{i k l}\left\{\delta x_{k}^{(1)}, \delta x_{l}^{(1)}\right\}\right],
\end{aligned}
$$

so that by using (20), (62), and (63) we arrive at

$$
\ddot{\eta}_{i}^{(2)}+\omega_{3}^{2} \eta_{i}^{(2)}=u_{0}^{2} T_{i k} \eta_{k}^{(2)}+u_{0} Q_{i k} \eta_{k}^{(2)}+F_{i}^{(2)} .
$$

For simplicity, we have suppressed the spin indices $j$ and $m$ and have made all time dependence implicit in (65). Notice that the $n=2$ equations (65) are just the $n=1$ equations (21), driven by the forcing term $F_{i}^{2 j m}(t)$. The latter can be written as a bilinear form:

$$
F_{i}^{2 \gamma}(t)=\eta_{k}^{1 \alpha} K_{i k l ; \alpha \beta}^{\gamma} \eta_{l}^{1 \beta},
$$

where once more we denote $\alpha \equiv j m, \beta \equiv j^{\prime} m^{\prime}$, and $\gamma \equiv$ $j^{\prime \prime} m^{\prime \prime}$ and have omitted the sums on the spatial indices $k$ and $l$ and the spin indices $\alpha$ and $\beta$. The matrix of the bilinear form $K$ is given by

$$
\begin{aligned}
K_{i k l ; \alpha \beta}^{\gamma}= & \left(\mathcal{J}_{a}\right)_{\dot{m} m}^{(j)} f_{j \dot{m}, \beta}^{\gamma}\left(\epsilon_{b a k} \epsilon_{b i l}+\epsilon_{b a i} \epsilon_{b k l}\right) \\
& +\frac{1}{2} \epsilon_{i k l} f_{\alpha \beta}^{\gamma},
\end{aligned}
$$

where $\mathcal{J}_{a} \equiv-i u_{0} J_{a}$ and we have omitted the dependence of $\eta_{i}^{1 j m}(t)$ on the dimensionless time $t \equiv \mu \tau$. As usual, we have also omitted the sums in the spatial indices $a$ and $b$ and the spin index $\dot{m}$ in (67). In compact form, (65) can be written as 


$$
\ddot{H}^{(2)}+\mathcal{K} \cdot H^{(2)}=F^{(2)}, \quad F^{(2)} \equiv H^{(1)} K H^{(1)} .
$$

Let us now discuss the role of the LO unstable modes. The presence of unstable LO modes [i.e., having $\lambda_{-}^{2}<0$ in (40)] in the forcing (67) can induce further unstable modes at the NLO, for any spin $j$. The form of the structure constants $f_{1 m, \beta}^{\gamma}$ and $f_{2 m, \beta}^{\gamma}$ [cf. (A2)-(A5) in the Appendix] implies that the coupling of an unstable LO mode with $j=1$ to a stable LO mode $j^{\prime}$ can destabilize all the $j^{\prime \prime}=j^{\prime}$ modes at NLO order. Similarly, the coupling of an unstable LO mode with $j=2$ to a stable LO mode $j^{\prime}$ can destabilize all the $j^{\prime \prime}=j^{\prime} \pm 1$ modes at NLO order. The cascade of perturbative instabilities extends to the higher perturbative orders, so that all the modes at any given perturbative order $n$ can become unstable because of the LO instabilities at $j=1,2$.

Another possible source of instabilities shows up whenever the frequency of the external forcing $F_{i}^{2 \gamma}(t)$ in (67) matches one of the system's natural eigenfrequencies (39)-(41). These resonances are not restricted to the unstable critical point $\mathbf{u}_{1 / 6}$ but they may also destabilize the stable critical point $\mathbf{u}_{1 / 3}$ (i.e., the Myers sphere). For example, a resonance will occur when a zero mode in the $P$ sector couples to a mode in the $R_{ \pm}$sectors in such a way that the resulting forcing (67) contains at least one of the system's natural eigenfrequencies (39)-(41). The above discussion has to take into consideration the LO and NLO Gauss law constraints which impose the symmetries to the solution of the equations.

\section{B. Gauss law constraint (NLO)}

The LO constraint equation (59) implies that the initial conditions for the velocity of any mode $\eta_{i}^{(1)}$ must be orthogonal to the $P$ sector and thus it should be a linear combination of the eigenvectors of the other two sectors $R_{ \pm}$. The NLO Gauss law constraint (17) reads

$$
\left\{\dot{x}_{i}^{(2)}, x_{i}^{(0)}\right\}+\left\{\dot{x}_{i}^{(1)}, x_{i}^{(1)}\right\}=0,
$$

since $\dot{x}_{i}^{(0)}=0$. Substituting the values of $x_{i}^{(0)}$ and $\dot{x}_{i}^{(n)}$ from (7) and (18) we obtain

$$
u_{0} \dot{\eta}_{i}^{2 j m}\left\{Y_{j m}, e_{i}\right\}+\dot{\eta}_{i}^{1 j m} \eta_{i}^{1 j^{\prime} m^{\prime}}\left\{Y_{j m}, Y_{j^{\prime} m^{\prime}}\right\}=0,
$$

where the sums over all the repeated indices have been omitted as usual. Calculating the brackets by using (20) and (63) it follows that

$$
i u_{0} \dot{\eta}_{i}^{2 j m}\left(J_{i}\right)_{m^{\prime} m}^{(j)} Y_{j m^{\prime}}+\dot{\eta}_{i}^{1 j m} \eta_{i}^{1 j^{\prime} m^{\prime}} f_{j m, j^{\prime} m^{\prime}}^{j^{\prime \prime} m^{\prime \prime}} Y_{j^{\prime \prime} m^{\prime \prime}}=0,
$$

which gives

$$
i u_{0} \dot{\eta}_{i}^{2 j^{\prime \prime} m}\left(J_{i}\right)_{m^{\prime \prime} m}^{\left(j^{\prime \prime}\right)}+\dot{\eta}_{i}^{1 j m} \eta_{i}^{1 j^{\prime} m^{\prime}} f_{j m, j^{\prime} m^{\prime}}^{j^{\prime \prime} m^{\prime \prime}}=0
$$

after factoring out the spherical harmonics. This equation implies that the initial velocity $\dot{\eta}_{i}^{(2)}$ must be a linear combination of the $R_{ \pm}$sectors and a component that belongs to the $P$ sector and is given by the LO forcing term that appears in the constraint. The latter depends on the coupling of the initial velocity $\dot{\eta}_{i}^{(1)}$ and the values of $\eta_{i}^{(1)}$ for different spins $j$.

\section{General solution}

The general solution of the NLO perturbation equations (68) can be written as the sum of the general solution of the homogeneous equation (24) and a special solution of the full equation (68):

$$
H^{(2)}(t)=H_{\mathrm{h}}^{(2)}(t)+H_{\mathrm{s}}^{(2)}(t)
$$

We have already shown that the general solution $H_{\mathrm{h}}^{(2)}(t)$ of the homogeneous equation (24) takes the form (42). By writing $\mathcal{K} \equiv \omega_{3}^{2}-u_{0}^{2} T-u_{0} Q=\Omega_{0}^{2}$, it is easy to see that $H_{\mathrm{h}}^{(2)}(t)$ can also be written as

$$
H_{\mathrm{h}}^{(2)}(t)=H_{\mathrm{h}}^{(2)}(0) \cos \Omega_{0} t+\dot{H}_{\mathrm{h}}^{(2)}(0) \Omega_{0}^{-1} \sin \Omega_{0} t .
$$

Similarly, the special solution $H_{\mathrm{s}}^{(2)}(t)$ takes the following form:

$$
\begin{aligned}
H_{\mathrm{s}}^{(2)}(t)= & \Omega_{0}^{-1} \sin \left(\Omega_{0} t\right) \int_{0}^{t} d s \cos \left(\Omega_{0} s\right) F^{(2)}(s) \\
& -\Omega_{0}^{-1} \cos \left(\Omega_{0} t\right) \int_{0}^{t} d s \sin \left(\Omega_{0} s\right) F^{(2)}(s) .
\end{aligned}
$$

Interestingly, the structure of the complete solution (75) remains the same at all higher perturbative orders $n=2,3, \ldots$, while it turns out that the corresponding forcing terms $F^{(n)}(s)$ depend on the solutions of the previous orders.

\section{EXAMPLE}

We now work out a simple example that demonstrates the instability cascade phenomenon. Let us compute $F_{i}^{2 \gamma}$ at the point $u_{0}=1 / 6$ and spin $j^{\prime \prime}=3$, when only the LO mode $\xi_{-}^{2,0} \equiv \xi$ is turned on. Then (42) becomes

$$
H(t)=e^{i \lambda_{-} t} \xi_{-}, \quad \xi_{-}=\left.\xi \cdot|-\rangle\right|_{j=2, m=0} .
$$

Computing the value (53) of the eigenvector $|-\rangle$ for $j=2$ and $m=0$ it is easy to show that only the following $j=2$ components of $\eta_{i}^{1 \alpha}$ are nonzero:

$$
\eta_{x}^{1,2, \pm 1}= \pm \frac{\xi}{2} \sqrt{\frac{3}{5}} \cdot e^{t /(3 \sqrt{2})}
$$




$$
\begin{aligned}
\eta_{y}^{1,2, \pm 1} & =-\frac{i \xi}{2} \sqrt{\frac{3}{5}} \cdot e^{t /(3 \sqrt{2})} \\
\eta_{z}^{1,2,0} & =-\xi \sqrt{\frac{2}{5}} \cdot e^{t /(3 \sqrt{2})} .
\end{aligned}
$$

It can be shown that the solution (77)-(79) satisfies both the reality condition (19) and the LO Gauss constraint (59). Plugging (77)-(79) into the expression (67) for the NLO forcing term, we obtain the following nonzero components:

$$
\begin{aligned}
F_{x}^{2,3, \pm 1} & = \pm \frac{2 \xi^{2}}{5} \sqrt{\frac{3}{7 \pi}} \cdot e^{\sqrt{2} t / 3}, \\
F_{y}^{2,3, \pm 1} & =-\frac{2 i \xi^{2}}{5} \sqrt{\frac{3}{7 \pi}} \cdot e^{\sqrt{2} t / 3}, \\
F_{z}^{2,3,0} & =-\frac{6 \xi^{2}}{5 \sqrt{7 \pi}} \cdot e^{\sqrt{2} t / 3},
\end{aligned}
$$

by using the analytic expressions (A3)-(A5) for the structure constants $f_{\alpha \beta}^{\gamma}$ and the well-known angular momentum matrices $J_{i}$ (in the spin- $j$ representation). The parametric plot of the forcing (80)-(82) can be found in Fig. 1. Because the only nonzero components of the LO modes are (77)-(79), we only need the following structure constants:

$$
\begin{aligned}
f_{2,0 ; 2,1}^{3,1} & =-f_{2,1 ; 2,0}^{3,1}=-f_{2,0 ; 2,-1}^{3,-1}=f_{2,-1 ; 2,0}^{3,-1}=-3 i \sqrt{\frac{2}{7 \pi}}, \\
f_{2,1 ; 2,-1}^{3,0} & =-f_{2,-1 ; 2,1}^{3,0}=\frac{6 i}{\sqrt{7 \pi}} .
\end{aligned}
$$

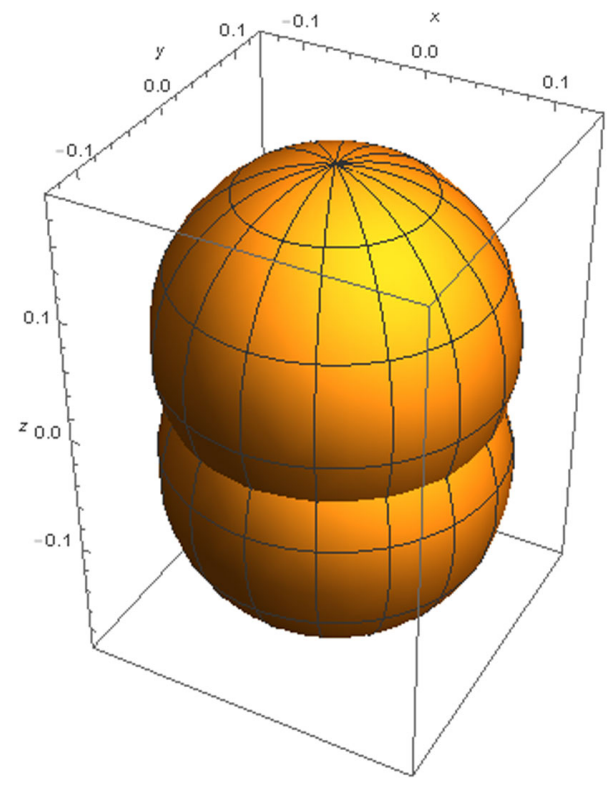

FIG. 1. Parametric plot of the forcing (80)-(82).
We next insert the expression for the forcing (80)-(82) into the $n=2$ perturbation equation (65) which we want to solve for the mode $\eta_{i}^{2,3, m}$ that was metastable for $n=1$ [cf. Eq. (40)]. As usual, the general solution of (65) can be cast in the following form:

$$
\eta_{k}^{2 \gamma}(t)=\tilde{\eta}_{k}^{\gamma}(t)+\zeta_{k}^{\gamma} e^{\sqrt{2} t / 3}, \quad k=1,2,3,
$$

where $\tilde{\eta}_{k}^{\gamma}(t)$ is the general solution of the homogeneous part (24) of (68) and is thus given by a formula of the form (42), whereas $\zeta_{k}^{\gamma}$ is a special solution. Plugging (84) into (65) and using the fact that $\tilde{\eta}_{k}^{\gamma}(t)$ solves the homogeneous equation (21), we get

$$
\left(\frac{2}{9}+\omega_{3}^{2}\right) \zeta_{i}-u_{0}\left(u_{0} T_{i k}+Q_{i k}\right) \zeta_{k}=\tilde{f}_{i}
$$

where we have set $F_{k}^{2 \gamma} \equiv \tilde{f}_{k}^{\gamma} e^{\sqrt{2} t / 3}$ in (80)-(82) so that the coefficients $\tilde{f}_{k}^{\gamma}$ can be directly read off from the forcing. It is easy to work out the solution of (85):

$$
\begin{aligned}
\zeta_{x}^{3, \pm 1} & = \pm \frac{9 \xi^{2}}{5} \sqrt{\frac{3}{7 \pi}} \\
\zeta_{y}^{3, \pm 1} & =-\frac{9 i \xi^{2}}{5} \sqrt{\frac{3}{7 \pi}} \\
\zeta_{z}^{3,0} & =-\frac{27 \xi^{2}}{5 \sqrt{7 \pi}}
\end{aligned}
$$

The NLO initial conditions (60) imply

$$
\eta_{k}^{2 \gamma}(0)=\dot{\eta}_{k}^{2 \gamma}(0)=0,
$$

which leads to the following set of constraints for the solution of the homogeneous equation $\tilde{\eta}_{k}^{\gamma}(t)$ :

$$
\begin{array}{ll}
\tilde{\eta}_{x, y}^{3, \pm 1}(0)=-\zeta_{x, y}^{3, \pm 1}, & \tilde{\eta}_{z}^{3,0}(0)=-\zeta_{z}^{3,0}, \\
\dot{\tilde{\eta}}_{x, y}^{3, \pm 1}(0)=-\frac{\sqrt{2}}{3} \zeta_{x, y}^{3, \pm 1}, & \dot{\tilde{\eta}}_{z}^{3,0}(0)=-\frac{\sqrt{2}}{3} \zeta_{z}^{3,0} .
\end{array}
$$

Based on the above, it can be shown that the solution (84) satisfies the NLO Gauss law constraint (72) at the initial time $t=0$.

The coupling of the $j=2$ modes leads to a nonvanishing forcing $F_{i}^{2 \gamma}$, not only in the case $j^{\prime \prime}=3$ (which we have just treated), but also when the spin becomes $j^{\prime \prime}=1$. Plugging (77)-(79) into the expression (67) for the NLO forcing term, we obtain the following nonzero forcing components:

$$
F_{x}^{2,1,1, \pm 1}= \pm \frac{3 \xi^{2}}{40} \sqrt{\frac{3}{2 \pi}} \cdot e^{\sqrt{2} t / 3}
$$




$$
\begin{aligned}
F_{y}^{2,1, \pm 1} & =-\frac{2 i \xi^{2}}{40} \sqrt{\frac{3}{2 \pi}} \cdot e^{\sqrt{2} t / 3} \\
F_{z}^{2,1,0} & =-\frac{\xi^{2}}{10} \sqrt{\frac{3}{\pi}} \cdot e^{\sqrt{2} t / 3}
\end{aligned}
$$

It is again straightforward to calculate the special solution of (85):

$$
\begin{gathered}
\zeta_{x}^{1, \pm 1}= \pm \frac{29 \xi^{2}}{20 \sqrt{6 \pi}} \\
\zeta_{y}^{1, \pm 1}=-\frac{29 i \xi^{2}}{20 \sqrt{6 \pi}} \\
\zeta_{z}^{1,0}=-\frac{8 \xi^{2}}{5 \sqrt{3 \pi}}
\end{gathered}
$$

The NLO initial conditions (60) can again be enforced by choosing an appropriate solution $\tilde{\eta}_{k}^{\gamma}(t)$ of the homogeneous equation (24).

It is interesting to take a closer look at the decomposition of the above solutions into the three sectors $P$ and $R_{ \pm}$. As we have already mentioned, the LO mode $\xi_{-}^{2,0} \equiv \xi$ that we turned on belongs to the $R_{-}$sector and corresponds to $j=2$ and $m=0$. It is a matter of algebra to show that the solutions (86)-(88) and (95)-(97) can be expressed in the $3 \times(2 j+1)$-dimensional space as follows:

$$
Z_{3}=\left.\frac{9 \xi^{2}}{5} \sqrt{\frac{3}{\pi}} \cdot|-\rangle\right|_{j=3, m=0}
$$

and

$$
Z_{1}=-\left.\frac{\xi^{2}}{10 \sqrt{2 \pi}} \cdot|+\rangle\right|_{j=1, m=0}+\left.\frac{3 \xi^{2}}{2 \sqrt{\pi}} \cdot|-\rangle\right|_{j=1, m=0},
$$

where we defined the $3 \times(2 j+1)$ vectors

$$
Z_{1}=\left(\begin{array}{c}
\zeta_{x}^{1 m} \\
\zeta_{y}^{1 m} \\
\zeta_{z}^{1 m}
\end{array}\right), \quad Z_{3}=\left(\begin{array}{c}
\zeta_{x}^{3 m} \\
\zeta_{y}^{3 m} \\
\zeta_{z}^{3 m}
\end{array}\right)
$$

Therefore the instabilities not only propagate to NLO modes of higher angular momenta $j$ (compared to the LO instabilities), but also between sectors. Note however that the magnitude of the $R_{+}$instability is suppressed since $\left|\zeta_{-}^{3}\right| \simeq 44\left|\zeta_{+}^{1}\right|$ and $\left|\zeta_{-}^{1}\right| \simeq 21\left|\zeta_{+}^{1}\right|$.

\section{CONCLUSIONS AND DISCUSSION}

Motivated by the idea that the dynamics of the microscopic degrees of freedom on the horizon of static spherically symmetric black holes can be described by the BMN matrix model (a highly nonlocal field theory), we study the chaotic properties of this theory's classical continuum limit, that is, super M2-brane theory in the background (1)-(2). For previous work on this topic see [21]. The solution (1)-(2) is nothing more that the Penrose-Güven $[10,22]$ limit of the maximally supersymmetric backgrounds $\operatorname{AdS}_{7,4} \times S^{4,7}$ of 11-dimensional supergravity. Because of the flux term (2), the corresponding supermembrane theory is found to contain both stable and unstable sectors. In the present paper we study the NLO perturbative dynamics of classical solutions of spherical topology in the $S O(3)$ sector of the continuum limit of the BMN matrix model.

We show that the LO instabilities of certain spherical solutions (which appear exclusively in the dipole $j=1$ and quadrupole $j=2$ sectors of multipole perturbations; see [7]) give rise to a cascade of NLO instabilities that extend to all multipole sectors. The transmission of instabilities is due to the nonlinear coupling of NLO perturbations, which is in turn induced by the infinitedimensional area-preserving symmetry of the membrane. The instability cascade is further enhanced at subsequent perturbative orders. The physical reason behind the development of instabilities is related to the infinitedimensional symmetry of relativistic membranes. In other words, the development of infinitely many thin tubes only costs an infinitesimal amount of energy to the membrane. These thin tubes correspond to large values of the angular momentum quantum number $j$ arising in the expansion of perturbations in the multipole basis. This confirms our expectations that the unstable solutions ultimately induce a hedgehog structure on the membrane. In order to regularize these instabilities one has to rely on the matrix model truncation of the membrane (i.e., the BMN matrix model) where the dimension of the matrices $N$ determines a maximum value for each $j<N-1$.

Concerning the chaotic properties of BMN membranes, the matrix model has a higher degree of nonlocality and is therefore expected to scramble perturbations faster than the continuous membrane (which contains a local Poisson bracket interaction term). Another exciting aspect relating to the development of membrane instabilities is the possibility of topology changes that are caused by the self-interactions of the membranes. This is a long-standing problem in the field and is closely related to our understanding of membrane field theory. Presently, only Euclidean-time solutions have been shown to be able to induce topology changes in membranes (see e.g., [23,24]). Because matrix theory is a field theory of discrete membranes (i.e., seen as bound states of D0-branes [13]) it stands much better chances of providing an answer to this riddle.

A straightforward extension of our present work is the study of NLO perturbations of the BMN membrane in the $S O(3) \times S O(6)$ sector. The $S O(6)$ rotating configuration encodes the effect of higher dimensions on the $3+1$ dimensional fluid system. 


\section{ACKNOWLEDGMENTS}

The authors would like to thank C. Bachas, C. Efthymiopoulos, J. Hoppe, J. Iliopoulos and S. Nicolis for illuminating discussions. The present research is funded in the context of the project "Chaotic dynamics and black holes in BMN theory" E-12386 (MIS 5047794) under the call for proposals "Supporting researchers with an emphasis on young researchers-Cycle B" (EDULLL 103). The project is cofinanced by Greece and the European Union (European Social Fund-ESF) by the Operational Programme Human Resources Development, Education and Lifelong Learning 2014-2020.

\section{APPENDIX: STRUCTURE CONSTANTS}

The structure constants $f_{\alpha \beta}^{\gamma}$ of spherical harmonics that show up in the forcing term (67) were defined in (63). It is rather straightforward to invert (63):

$$
f_{\alpha \beta}^{\gamma}=\int_{\mathrm{S}^{2}} Y_{\gamma}^{*}(\theta, \phi)\left\{Y_{\alpha}(\theta, \phi), Y_{\beta}(\theta, \phi)\right\} d \Omega
$$

in order to obtain a closed formula for $f_{\alpha \beta}^{\gamma}$ that is valid for all the values of the quantum numbers $\alpha \equiv j m, \beta \equiv j^{\prime} m^{\prime}$ and $\gamma \equiv j^{\prime \prime} m^{\prime \prime}$. In the present paper, we only need the first few of them (see also [25]):

$$
\begin{aligned}
f_{1, \pm 1 ; j m}^{j^{\prime} m^{\prime}}= & \pm i \sqrt{\frac{3}{8 \pi}} \cdot \sqrt{(j \mp m)(j \pm m+1)} \delta_{j^{\prime} j} \delta_{m^{\prime}, m \pm 1}, \quad f_{1,0 ; j m}^{j^{\prime} m^{\prime}}=-i m \cdot \sqrt{\frac{3}{4 \pi}} \delta_{j^{\prime} j} \delta_{m^{\prime} m}, \\
f_{2,0 ; j m}^{j^{\prime} m^{\prime}}= & -3 i m \sqrt{\frac{5}{4 \pi}} \cdot\left[\sqrt{\frac{(j+1)^{2}-m^{2}}{(2 j+1)(2 j+3)}} \delta_{j^{\prime}, j+1}+\sqrt{\frac{j^{2}-m^{2}}{(2 j+1)(2 j-1)}} \delta_{j^{\prime}, j-1}\right] \cdot \delta_{m^{\prime} m}, \\
f_{2, \pm 1 ; j m}^{j^{\prime} m^{\prime}}= & \pm i \sqrt{\frac{15}{8 \pi}} \cdot\left[(j \mp 2 m) \cdot \sqrt{\frac{(j \pm m+1)(j \pm m+2)}{(2 j+1)(2 j+3)}} \delta_{j^{\prime}, j+1}\right. \\
& +(j \pm 2 m+1) \cdot \sqrt{\left.\frac{(j \mp m-1)(j \mp m)}{(2 j+1)(2 j-1)} \delta_{j^{\prime}, j-1}\right]} \cdot \delta_{m^{\prime}, m \pm 1}, \\
f_{2, \pm 2 ; j m}^{j^{\prime} m^{\prime}}= & \pm i \sqrt{\frac{15}{8 \pi}} \cdot\left[\sqrt{\frac{(j \mp m)(j \pm m+1)(j \pm m+2)(j \pm m+3)}{(2 j+1)(2 j+3)}} \delta_{j^{\prime}, j+1}\right. \\
& \left.-\sqrt{\frac{(j \mp m)(j \mp m-1)(j \mp m-2)(j \pm m+1)}{(2 j+1)(2 j-1)}} \delta_{j^{\prime}, j-1}\right] \cdot \delta_{m^{\prime}, m \pm 2} .
\end{aligned}
$$

Equation (A1) implies that the structure constants $f_{\alpha \beta}^{\gamma}$ obey the following sum rules:

$$
m+m^{\prime}=m^{\prime \prime}, \quad j+j^{\prime}+j^{\prime \prime}=\text { odd },
$$

where the second equation is obtained by setting $(\theta, \phi) \rightarrow$ $(\pi-\theta, \phi+\pi)$ in (A1). In addition $j, j^{\prime}$ and $j^{\prime \prime}$ obey triangle inequalities, e.g.,

$$
\left|j-j^{\prime}\right|+1 \leq j^{\prime \prime} \leq\left|j+j^{\prime}\right|-1,
$$

and its cyclic permutations hold. For the purposes of our work, this implies that when modes $n^{1 j m}$ are turned on up to $j_{\max }$, the forcing term vanishes for $F^{2 j^{\prime \prime} m^{\prime \prime}}$ for $j^{\prime \prime} \geq 2 j_{\max }$.
[1] Y. Sekino and L. Susskind, Fast scramblers, J. High Energy Phys. 10 (2008) 065.

[2] T. Damour, Black hole eddy currents, Phys. Rev. D 18, 3598 (1978).

[3] K. S. Thorne, R. H. Price, and D. A. Macdonald, Black Holes: The Membrane Paradigm (Yale University Press, 1986).
[4] J. Hoppe, Quantum theory of a massless relativistic surface and a two-dimensional bound state problem, Ph.D. thesis, Massachusetts Institute of Technology, 1982.

[5] K. Dasgupta, M. M. Sheikh-Jabbari, and M. Van Raamsdonk, Matrix perturbation theory for M-theory on a pp-wave, J. High Energy Phys. 05 (2002) 056. 
[6] M. Axenides, E. Floratos, and G. Linardopoulos, M2-brane dynamics in the classical limit of the BMN matrix model, Phys. Lett. B 773, 265 (2017).

[7] M. Axenides, E. Floratos, and G. Linardopoulos, Multipole stability of spinning M2 branes in the classical limit of the BMN matrix model, Phys. Rev. D 97, 126019 (2018).

[8] M. Axenides, E. Floratos, D. Katsinis, and G. Linardopoulos, M-Theory as a Dynamical System Generator, arXiv:2007.07028 [Springer Proceedings in Complexity (to be published)].

[9] U. Frisch, Turbulence: The Legacy of A. N. Kolmogorov (Cambridge University Press, Cambridge, England, 1995).

[10] R. Penrose, Any space-time has a plane wave as a limit, in Differential Geometry and Relativity. A Volume in Honour of André Lichnerowicz on His 60th Birthday, edited by M. Cahen and M. Flato, Mathematical Physics and Applied Mathematics Vol. 3 (Springer, Dordrecht, 1976), p. 271.

[11] J. M. Figueroa-O'Farrill and G. Papadopoulos, Maximally supersymmetric solutions of ten-dimensional and elevendimensional supergravities, J. High Energy Phys. 03 (2003) 048.

[12] D. Berenstein, J. Maldacena, and H. Nastase, Strings in flat space and pp waves from $\mathcal{N}=4$ super Yang-Mills, J. High Energy Phys. 04 (2002) 013.

[13] T. Banks, W. Fischler, S. H. Shenker, and L. Susskind, M-theory as a matrix model: A conjecture, Phys. Rev. D 55, 5112 (1997).

[14] R. C. Myers, Dielectric branes, J. High Energy Phys. 12 (1999) 022.
[15] T. Harmark and K. G. Savvidy, Ramond-Ramond field radiation from rotating ellipsoidal membranes, Nucl. Phys. B585, 567 (2000).

[16] P. A. Collins and R. W. Tucker, Classical and quantum mechanics of free relativistic membranes, Nucl. Phys. B112, 150 (1976).

[17] K. Efstathiou and D. Sadovskií, Perturbations of the $1: 1: 1$ resonance with tetrahedral symmetry: A three degree of freedom analogue of the two degree of freedom HénonHeiles Hamiltonian, Nonlinearity 17, 415 (2004).

[18] S. Lefschetz, Differential Equations: Geometric Theory (Wiley, New York, 1957).

[19] M. Axenides, E. G. Floratos, and L. Perivolaropoulos, Quadrupole instabilities of relativistic rotating membranes, Phys. Rev. D 64, 107901 (2001).

[20] M. E. Rose, Elementary Theory of Angular Momentum (Reidel, Dordrecht, 1957).

[21] Y. Asano, D. Kawai, and K. Yoshida, Chaos in the BMN matrix model, J. High Energy Phys. 06 (2015) 191.

[22] R. Güven, Plane-wave limits and T-duality, Phys. Lett. B 482, 255 (2000).

[23] D. Berenstein, E. Dzienkowski, and R. Lashof-Regas, Spinning the fuzzy sphere, J. High Energy Phys. 08 (2015) 134.

[24] S. Kovacs, Y. Sato, and H. Shimada, On membrane interactions and a three-dimensional analog of Riemann surfaces, J. High Energy Phys. 02 (2016) 050.

[25] T. A. Arakelian and G. K. Savvidy, Geometry of a group of area-preserving diffeomorphisms, Phys. Lett. B 223, 41 (1989). 